\title{
PRODUCTS
}
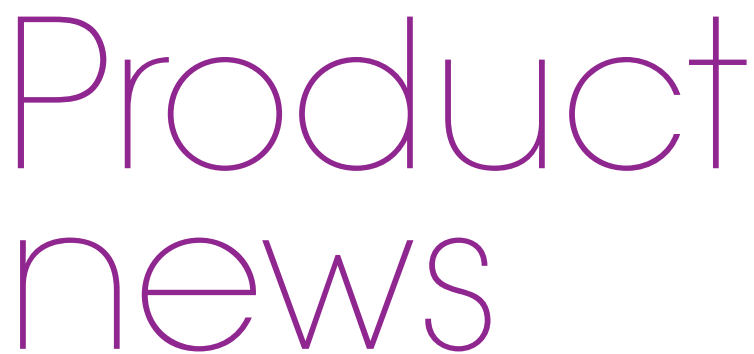

\section{EVERYONE CAN HAVE FRESH BREATH IN 2016}

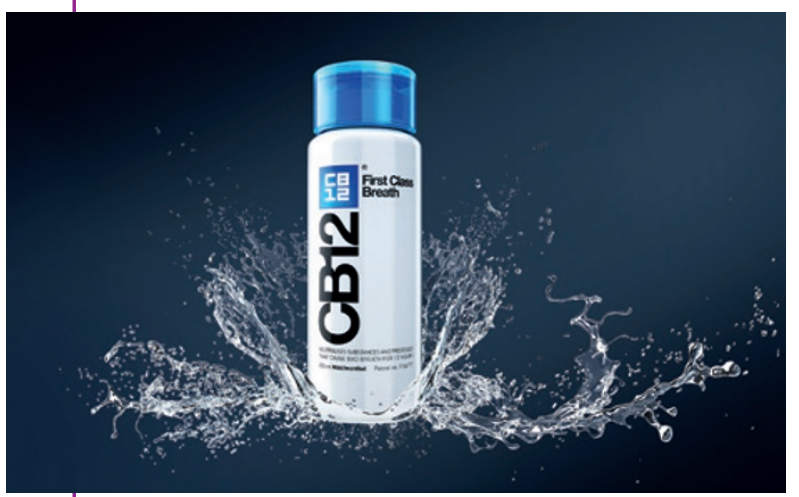

targeting and neutralising the volatile sulphur compounds that cause unpleasant breath. The active ingredients in CB12 also chemically bind to the tissues in the oral cavity to prevent bad breath for 12 hours.

CB12 contains $0.05 \%$ sodium fluoride to further encourage good oral health, and is available in mint menthol and mild mint menthol flavours to give your patients fresh breath confidence every day.

CB12 also offer sugar-free Boost

Bring confidence to your patients in 2016 by recommending CB12.

Many people lose their self-esteem either worrying about their breath or suffering with the embarrassment of oral malodour. A daily shot of CB12 mouthwash could change their lives for the better.

CB12 mouthwash works instantly by chewing gum with a pleasant mint flavour that can be used to boost breath confidence anywhere.

Help your patients make a new start this New Year by recommending CB12. To become a stockist or receive an information pack contact the team now on 01279873449 or visit www.cb12.co.uk.

\section{CUT DOWN ON PAPER FORM FILLING}

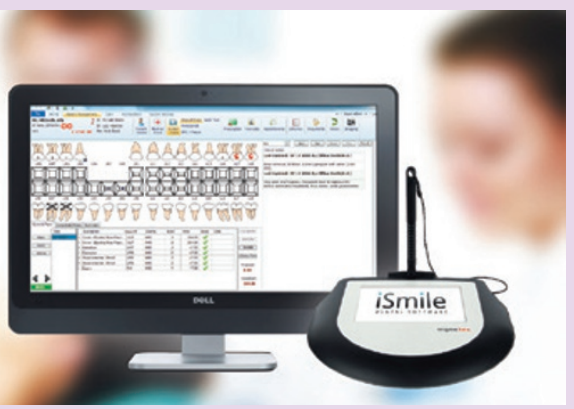

With more and more patients having access to smart phones and emails, it makes sense to extend your paperless system directly to your patient's mobile device. Patient Portal by iSmile allows your patients to fill out medical history forms and patient questionnaires on their mobile phones, tablets or desktop computers.

Forms are automatically emailed to patients, allowing them to securely fill them out online prior or post appointment. Not only does this give your patients a more connected experience, it also means less hassle filling out forms at reception.

Once the online forms are back in your practice management system they can be verified and signed by the patient using a digital signature pad. Patient Portal further allows you to use your own choice of tablet (iOS or Android) for form filling at the practice, in case there are patients that don't have access to a mobile device.

iSmile's powerful reporting system allows you to display data from patient feedback forms in a range of formats so you can analyse trends across different time.

www.ismiledental.co.uk

\section{SWIFT, EFFECTIVE} WHITENING AT HOME

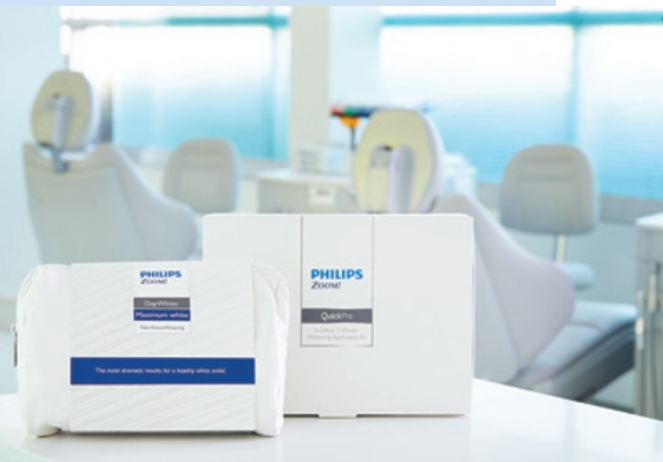

The Philips Zoom! QuickPro 6\% is the latest addition to the Philips Zoom! EU portfolio of whitening products and addresses the concerns voiced by many dental professionals as it offers convenience to the patient, without taking up valuable practice chair time or requiring custom made trays. It also fills the void left by the removal of over-thecounter tooth whitening products from the market. Philips Zoom! QuickPro 6\% is clinically proven to whiten up to four shades in as little as four days (average shade reduction; actual results

may vary).

No trays are needed and, provided the patient is judged suitable for treatment by a dentist, a dental professional simply walks through the first application with the patient and dispenses the product, saving valuable chair time.

\section{Philips Zoom! QuickPro can be} used as a stand-alone procedure: following a chairside treatment; or to 'top up' previous whitening. A general appointment can become a whitening appointment, thanks to the convenience of the five-minute application, making it easy and cost effective to add whitening to any appointment, especially routine hygiene sessions.

Many patients who used to buy OTC whitening products still want to whiten with the convenience of a home-use kit. Philips Zoom! QuickPro provides an affordable but professional whitening solution with that convenience.

More information is available from www.philips-tsp.co.uk/bdia. 